\title{
Prevalence and characteristics of non-lactate and lactate expressors in septic shock
}

\author{
Julie Mackenhauer ${ }^{1,2^{*}}$, Andrea Dugas ${ }^{1}$, Nina Joyce ${ }^{1}$, Michael W Donnino ${ }^{1}$ \\ From Danish Society for Emergency Medicine: Research Symposium 2010 \\ Roskilde, Denmark. 20-21 May 2010
}

\section{Background}

Although elevated lactate levels are associated with increased mortality in sepsis, not all patients with septic shock express lactic acidosis. The objective of this study was to determine the proportion of patients in vasopressor-dependent septic shock who presented with and without lactic acidosis. Secondarily, we sought to determine patient characteristics that differentiated lactate expressors and non-lactate expressors.

Hypothesis: Patients in septic shock often do not have an elevated lactate, and still face significant mortality.

\section{Methods}

Retrospective review of patients presenting between 01/ 08 and $09 / 08$, to an urban tertiary care emergency department (50,000 patients year). Patients with a presumed diagnosis of septic shock requiring vasopressors were divided, based on initial lactate, to low (0-2.4), intermediate (2.5 - 3.9) and high (4.0 and higher) lactate groups. We used descriptive statistics to report the data and chi-squared or t-test as appropriate to compare groups.

\section{Results}

A total of 138 patients with vasopressor-dependent shock were enrolled. Of these, 64/138 (46\%) were nonlactate expressors (low lactate levels), and $54 \%$ of the population were lactate expressors, with $33 / 138(24 \%)$ in the intermediate elevation group and $41 / 138(30 \%)$ in the high lactate group. The 28-day mortality increased significantly with increasing lactate with $19 \%, 30 \%$, and $44 \%$ mortality within the low, intermediate and high lactate group respectively $(\mathrm{p}=0.021)$. Acute liver failure statistically correlated with higher lactate levels with $4 \%$
(5/64), $16 \%(4 / 33)$, and $34 \%(15 / 41)$ prevalence as the lactate group increased from low, intermediate to high $(\mathrm{p}<0.001)$. Similarly, liver disease and liver enzymes trended in the same direction. Bacteremia increased significantly between lactate groups with $9 \%(6 / 64)$ in the normal lactate, $15 \%(5 / 33)$ in the intermediate lactate and $34 \%(14 / 41)$ in the high lactate group having positive blood cultures $(\mathrm{p}=0.005)$.

\section{Conclusion}

Almost one-half of patients with vasopressor-dependent septic shock do not express lactic acidosis on presentation, though a high mortality rate remains in this population. The use of lactic acidosis as the only indication of perfusion status or endpoint of resuscitation in septic shock may be inadequate. We found an association between lactate expressors and acute liver failure and the prevalence of positive blood cultures.

\section{Author details}

${ }^{1}$ Beth Israel Deaconess Medical Center, Emergency Department, Boston, USA. ${ }^{2}$ Research Center for Emergency Medicine, Aarhus University Hospital, Aarhus, Denmark.

Published: 17 September 2010

doi:10.1186/1757-7241-18-S1-O2

Cite this article as: Mackenhauer et al:: Prevalence and characteristics of non-lactate and lactate expressors in septic shock. Scandinavian Journal of Trauma, Resuscitation and Emergency Medicine 2010 18(Suppl 1):O2.

* Correspondence: thejuliemac@gmail.com

${ }^{1}$ Beth Israel Deaconess Medical Center, Emergency Department, Boston, USA Full list of author information is available at the end of the article 